\title{
IndUSTRy Perceptions of GraduAte AtTRIBUTE REQUIREMENTS FOR THE WORKPLACE
}

\author{
Donald S. Petkau \\ Department of Biosystems Engineering, University of Manitoba, Winnipeg, MB, R3T 5V6 \\ Don.Petkau@umanitoba.ca
}

\begin{abstract}
At the Faculty of Engineering at the University of Manitoba research has been undertaken to determine the level of student competency in the graduate attributes as set forth by the CEAB accreditation process. This study takes an alternative view and seeks to understand the current industry requirements for a new graduate employee based on the graduate attributes. It consisted of a questionnaire completed by two groups of engineers working in a major energy corporation in the Province of Manitoba. One group consisted of senior engineers with a minimum of 15 years experience while the other was of new graduates with at minimum 18 months of service. The groups were asked to complete a questionnaire on the level of competency they felt was required for new graduates entering the workplace. This paper describes the process and the analysis of the information. Results were compared with an assessment of a new graduate's competency levels. The information shows that while a student's competency levels at graduation may be lower than expected they still generally meet the requirements of the workplace. Information also shows that areas of concern are not in the technical areas but rather in the professional skills.
\end{abstract}

Keywords: Graduate attributes, Engineering requirements, Student perspective

\section{INTRODUCTION}

Engineering schools in Canada are required to assess graduates of their programs according to specified graduate attributes in order to gain accreditation. At the University of Manitoba the assessment involves direct methods (assessing coursework to the Canadian Engineering Accreditation Board (CEAB) graduate attributes) and indirect methods including surveys and forums. Typically most of the data is collected from students at time of graduation with a self assessment process. The specific purpose of this paper is to describe my $\mathrm{PhD}$ research which included an in-depth study of the engineering competencies required in an engineering workplace.
There is little information on how a newly graduated engineer's education relates to the workplace and what additional learning takes place subsequent to their formal education. A determination of these factors could lead to the better understanding of whether this knowledge could be incorporated into an engineering educational program. This may also determine that this knowledge is more suited to a training program undertaken in the workplace during the first years of an engineers' career.

Currently we use a series of graduate attributes as determined by the accreditation program in Canada (Engineers Canada 2011) [2] to help determine the qualifications of a graduating engineer. These attributes are a definition of qualifications for engineering graduates upon their completion of an accredited program. The question is how these attributes define the capabilities of a new graduate. There is an understanding that a new graduate is not considered a professional until they have completed a further four years of mentorship under the tutelage of an experienced professional engineer. They must first complete a number of stages of development as required by the Association of Professional Engineers and Geoscientists of the Province of Manitoba (APEGM). Engineering graduates must complete a four year term as an Engineer in Training (EIT) prior to receiving their professional engineering designation (P.Eng). This program includes performance of engineering work under the supervision of a professional engineer, individual development opportunities, and exams in engineering practice and ethics. This is an indication that engineering graduates must gain additional skills and knowledge in the workplace after graduation. These are not currently defined and it may be difficult to determine this additional knowledge as each engineering position within each organization will require a different knowledge basis.

What are the additional qualifications an engineering graduate must attain subsequent to their formal engineering education? How do these qualifications provide a link between the formal education of an engineer and the practice of a professional engineer?

The primary objective of this research was to define how the graduate attributes used in our engineering education programs link to the practice of professional engineering. 
The specific objectives to achieve this goal were:

1. identify the role of an expert or professional engineer

2. identify the role of a novice or newly graduated engineer

3. identify the knowledge of a newly graduated engineer with respect to the graduate attributes

4. identify the knowledge requirements of the workplace for a newly graduated engineer with respect to the graduate attributes

5. determine the link and/or gaps between these abilities and knowledge requirements of newly graduated engineers to that of a professional engineer.

This paper will summarize the results of the perception of senior engineers and new graduates engineers on the job requirements of new graduates with respect to the $\mathrm{CEAB}$ graduate attributes.

\section{RESEARCH METHODOLOGY}

\subsection{Background}

This study is a quantitative descriptive study characterizing engineering experiences within the work environment using descriptive statistics with a series of mixed method data collection tools. This study was of an exploratory nature using a sequential data collection method. The first stage was in the collection of engineering task related information using an open ended interview style. A total of three interviews were conducted with two sets of participants. The first set was a group of professional engineers in senior management positions. The second set of participants was a group of newly graduated engineers in their first three years of employment. The interviews were designed to gather information on the job tasks of each group of engineers. The senior engineers were also interviewed on the required job tasks of the newly graduated engineer.

The second data collection technique was the use of questionnaires. A total of three questionnaires were completed by each participant. The first one was on the aspects of engineering practice which were correlated to the participants' job tasks compiled from the interviews. The second questionnaire was on the graduate attributes and correlated engineering education to the job tasks of a newly graduated engineer. The third questionnaire was also on the graduate attributes but this one evaluated the strengths and weaknesses of the new graduate.

This study took place in the offices of Manitoba Hydro wh i c h is a Crown Corporation in the province of Manitoba. It is the province's major energy utility supplying electricity and natural gas to customers throughout Manitoba. It also supplies electricity to other markets in Canada and the mid-western United States. The corporation has more than 6000 employees and operates out of numerous locations in Winnipeg and throughout the province of Manitoba.

When selecting the appropriate participants it was important to maintain variability in the sample as well as to eliminate bias in the selection methods. The size of this corporation and number of engineering departments allowed for potential variability. The type of study would restrict the number of participants involved. In order to be able to generalize results participants were chosen from a number of engineering disciplines. Manitoba Hydro has a variety of engineering disciplines on staff. Another important factor for recruitment was the availability and cooperation of the participants. The main recruitment criteria were the years of professional experience.

\subsection{Participants}

There were two separate groups of participants in this research study. The first was a group of four recent graduates from the engineering program at the University of Manitoba. They were all enrolled in the EIT program at APEGM and are referred to as EIT's in this study. This was a diversified group with two electrical engineers, one mechanical engineer, and one civil engineer. They had graduated from their programs from between fourteen months earlier to the longest at three years earlier. One of the individuals had no previous engineering experience even in a summer job. The other three individuals had been working in engineering related summer jobs prior to graduation. Two had worked for Manitoba Hydro in the past and so had additional experience in regards to some of the orientation and cultural aspects of the corporation. Three of the four individuals were currently in or had gone through the EIT program at Manitoba Hydro. This program allows new graduates to spend six months working in a particular area of the corporation. At the completion of their six month term they choose another department for the following six months. The EIT's are allowed to complete up to four of these terms prior to their permanent placement. The one individual who had not entered through this EIT program had spent a summer term in a particular department and upon graduation continued in the same role as a permanent employee. This information is relevant to the study as the individuals in the EIT program spend the first two years of employment in a situation equivalent to four new positions. This affects their job role as they need additional training and orientation with each of these placements. This could affect this study as individuals are involved with more entry level activities rather than a steady progression to increased responsibilities. This should not have a major affect though as only one of the individuals is currently in the EIT program. All of the other three participants are in permanent placements with two of them already in the second year of their positions. 
The second set of participants was a group of professional engineers and are referred to as P.Eng's in this study. All of these individuals have spent their careers with Manitoba Hydro and have had their professional engineering status from between nine to twenty-two years. These engineers are all in supervisory positions. They are currently supervising EIT's or have done so on numerous occasions in the past. All four of the professional engineering participants were electrical engineers.

\subsection{Ethical And Confidentiality Considerations}

This is a study involving individuals employed by a large corporation. Anytime people are part of a study considerations are required to protect the individuals. This is especially important for this study as interview information is requested from the participants on their engineering tasks and how these tasks are performed. Another consideration is the fact that all participants are employed by the same corporation. Some of the participants in the pilot study had supervisory/employee relationships. It was important to protect information exchanged and to eliminate the prospect of any coercion to participate. These issues were dealt with through appropriate ethical approval procedures. Anonymity measures were undertaken with all data sources including individual interview, focus groups, and questionnaires. Participants were never identified and data was coded to strip any identification prior to any publication. Consideration was taken that there was no position of power between members of the senior engineering group and the junior engineering group. All ethical issues were covered by signed agreements between participants and the researcher. The protocol for the project was approved by the University's research ethics board.

A second issue of confidentiality for this project was of a corporate nature. Manitoba Hydro was concerned that information detrimental to the corporation expressed during an interview could be released by the researcher. An agreement was reached with a signed contract between the corporation and the researcher to protect both parties.

\subsection{Survey Instrument}

The survey document was modified from the survey used by Cicek [1] in a survey of fourth year students at the University of Manitoba. The intent was to use the same instrument used on graduating students to survey new engineers after a number of years on the job. The modifications to the instrument, shown in Figure 1, were in the wording of the document. Participants were asked to evaluate the level of educational knowledge required to complete their work tasks at the time of entry to the workforce. The senior participants were also asked to evaluate the new employees in the same manner. They were instructed to give a general evaluation of the new graduates that they had supervised.

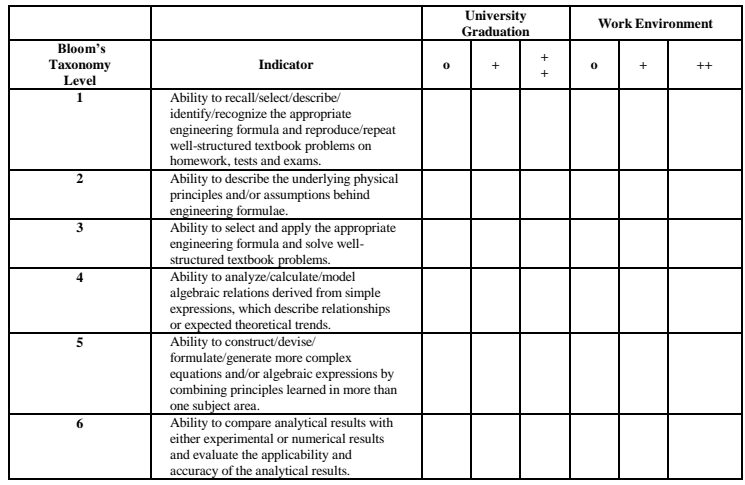

Figure 1: First page of Graduate Attribute Survey.

An additional survey instrument, shown in Figure 2, was also modified from one used by Cicek [1]. This asked the new graduates to rank the graduate attributes according to which was the strongest in their engineering education and which was the most important to the workplace. The senior participants were also asked to make this ranking based on their experience with new graduates and on which attribute was the most important for a new graduate upon entry to the workplace.

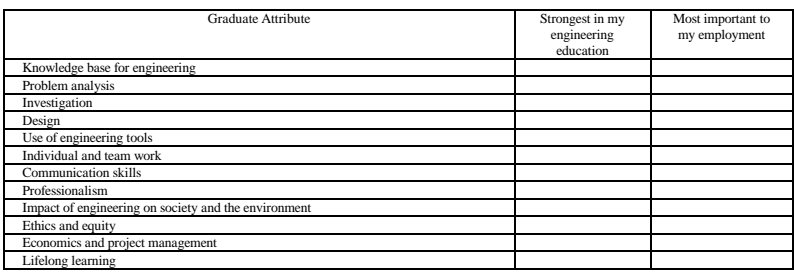

Figure 2: Last page of Graduate Attribute Survey for ranking attributes.

\section{RESULTS AND DISCUSSION}

\subsection{Results of EIT's Survey}

There were four EIT's which participated in both the survey and the interview portions of the study. A numerical value of " 1 " was assigned if the EIT's indicated that the skill/ability was not used in any of the work they were asked to complete, a value of " 2 " was assigned if the skill/ability was used but they needed support or additional training when completing these tasks and a value of " 3 " was assigned if the skill/ability was used in their work and they felt comfortable completing those tasks. The results of their perceived competencies required at time of entry to the workplace are summarized in Figure 3. 


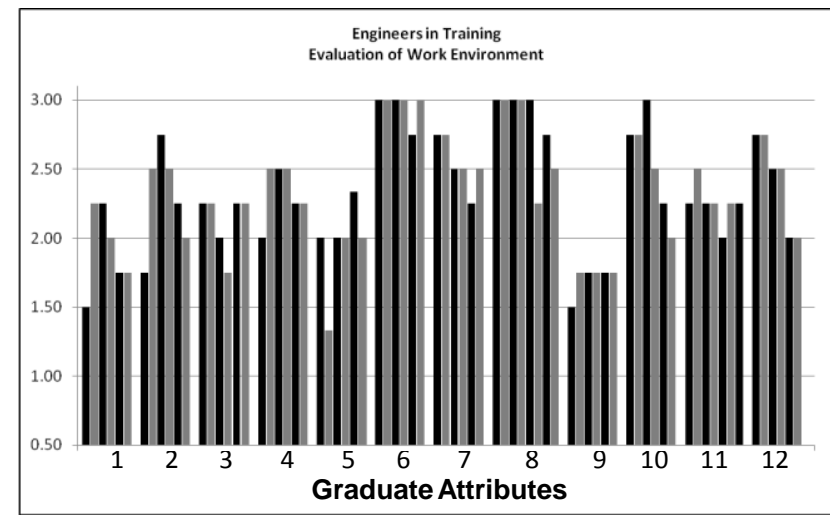

Figure 3: Average perceived graduate attribute workplace requirements for new engineering graduates by Engineers in Training.

The overall results show some substantial differences when compared to results from the evaluation of their own competencies at time of entry to the workplace. A major difference is that new graduates assessed an overall average value of 2.31 as the requirement for new graduates, compared to a value of 1.98 for their own competencies upon entering the workforce. This indicates that there were areas that these individuals struggled in completing tasks when they first entered the workplace. The results show that the participant's assessment of graduating students was that they have an average skill/ability level that would be considered only at the introduced level but that the requirements on the job were at a much higher level.

The other area of interest was in which of the attributes the EIT's considered to be the areas they lacked the skills/abilities needed for the job. In their own abilities the EIT participants rated the attributes of 2-Problem Analysis, and 3-Investigation as the highest for their skill/ability levels with an average rating of 2.38. The highest rated attributes for the work requirements were 6Individual and Team Work at a level of 2.96, 8Professionalism at a level of 2.75, along with 7Communication, and 10-Ethics and Equity at a rating of 2.54. In rating their own abilities the EIT's had a high rating of 2.38 for any one attribute which was in the technical area. While in the requirements they rated four attributes much high than their own abilities and these were all in the professional or soft skill areas. A second comparison is in the attributes that were rated at the low end of the scale. In the assessment of their own competencies the EIT's rated the lowest two attributes as 9- Impact on Society and Environment at 1.33 and 11Economics and Project Management at 1.63. There was some similarity here as the lowest attribute rating for required competency was again 9-Impact on Society and Environment at 1.71. The other attributes with low ratings were 1-Knowledge Base for Engineering, and 5Engineering Tools. While these had low ratings they came close to matching the competency level of the EIT's and therefore these participants felt that their competency levels were adequate for what the job required. In looking at the overall comparison between new graduates capabilities and the work requirements in Figure 4 we see that the EIT's felt their skill/ability levels matched the requirements in the first five attributes which cover the technical areas.

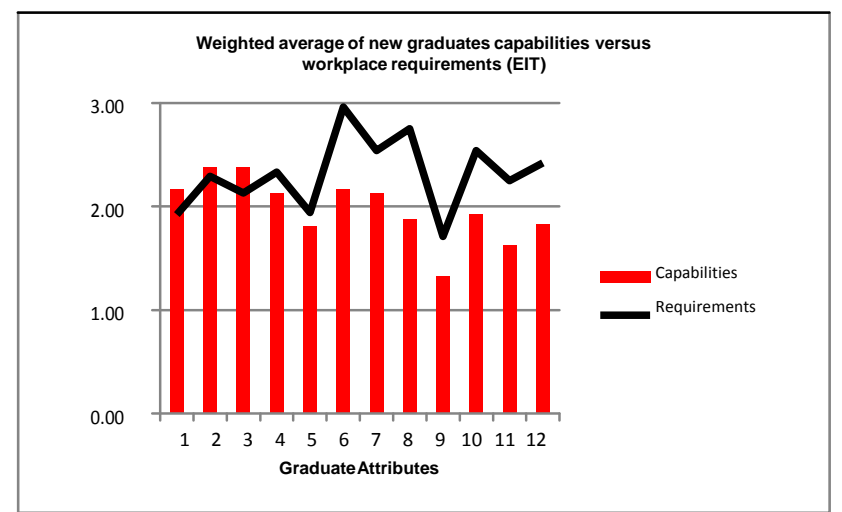

Figure 4: Average perceived graduate attribute workplace requirements for new engineering graduates by Engineers in Training.

It also shows that they felt their skill/abilities were lacking in the requirements of the remaining attributes. This information was reiterated in the individual interviews with discussions on how unprepared they were especially in the team work and project management areas.

Looking at each of the graduate attributes individually there are some interesting observations. After having used the survey based on Bloom's Taxonomy expectations were that skill/ability level would be highest in the lower levels and decrease with movement from the knowledge level to the evaluation level. This was not the case as in the attributes with high ratings, all six levels were rated high. In the technical attributes there was more varied distribution with the comprehension, application, and analysis levels receiving the highest ratings.

\subsection{Results of P.Eng Survey}

There were four P.Eng's which participated in both the survey and the interview portions of the study. A numerical value of "1" was assigned if the P.Eng indicated that the skill/ability was not used by a new graduate in any of the work they were asked to complete, a value of "2" was assigned if the skill/ability was used by a new graduate but they needed support or additional training when completing these tasks and a value of " 3 " was assigned if the skill/ability was used by a new graduate in the work they were asked to complete and they could complete it successfully. The results of their 
perceived competencies required at time of entry to the workplace are summarized in Figure 5.

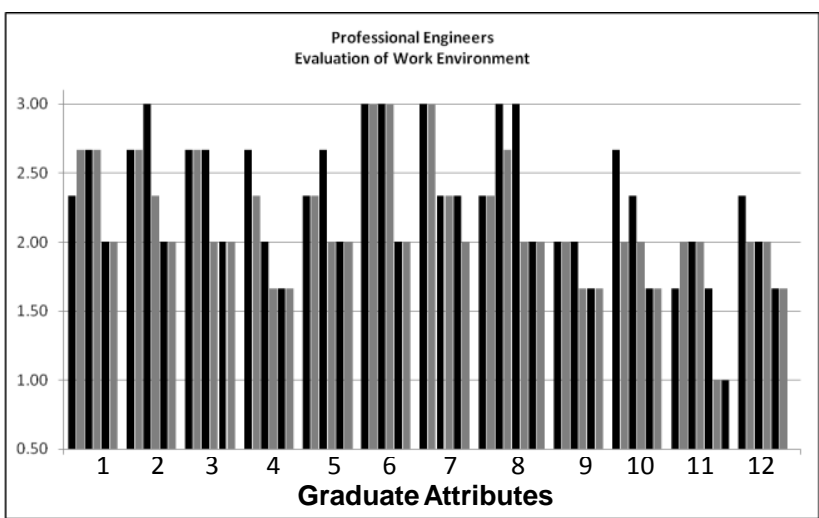

Figure 5: Average perceived graduate attribute workplace requirements for new engineering graduates by Professional Engineers.

The results from the P.Eng ratings (2.20) were similar to that of the EIT's (2.31). There were some similarities as the P.Eng's also rated 6-Individual and Team Work as the highest requirement at 2.67 and 7-Communication at 2.50. The lowest rated for the P.Eng participants was $\mathbf{1 1}$ Economics and Project Management at 1.61 which compares to a rating of 2.25 by the EIT's. This difference is explained through discussions in the interviews. The P.Eng's stated that project management was not a role required for new graduates and that they were satisfied with the competency of new employees. The EIT's felt that they were put into a project management role and that they were unprepared and uncomfortable with the expectations. The other attributes that the P.Eng's rated low were 9-Impact on Society and the Environment, and 12-Lifelong Learning. The senior group in rating new graduates coming into the workplace had a much more consistent ranking with respect to Blooms level. They consistently ranked the lower levels of knowledge and comprehension as high with the upper levels of synthesis and evaluation as much lower.

In looking at the overall comparison in Figure 6 we see that the P.Eng participants felt the EIT skill/ability levels at graduation matched the requirements more closely than the EIT self assessment.

There was a slight gap in skill/ability in attributes 2Problem Analysis and 3-Investigation and a more substantial gap in 6-Individual and Teamwork, 7Communication, and 8-Professionalism which were the same three with the largest gap indicated by the EIT's. All of the other attributes had the new graduates meeting the work requirements based on ratings by the P.Eng's. This shows agreement in both the senior and junior engineers what areas new graduates are lacking in skills and in which attributes they are meeting the job requirements.

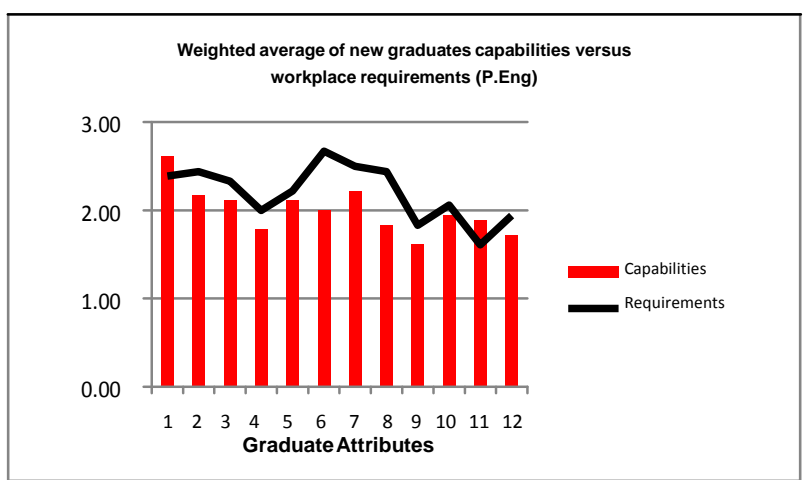

Figure 6: Average perceived graduate attribute workplace requirements for new engineering graduates by Professional Engineers.

\subsection{Comparing EIT to P.Eng Results}

Figure 7 shows a comparison between the P.Eng and the EIT participants on the skills/abilities required by a new graduate upon entry to the workplace.

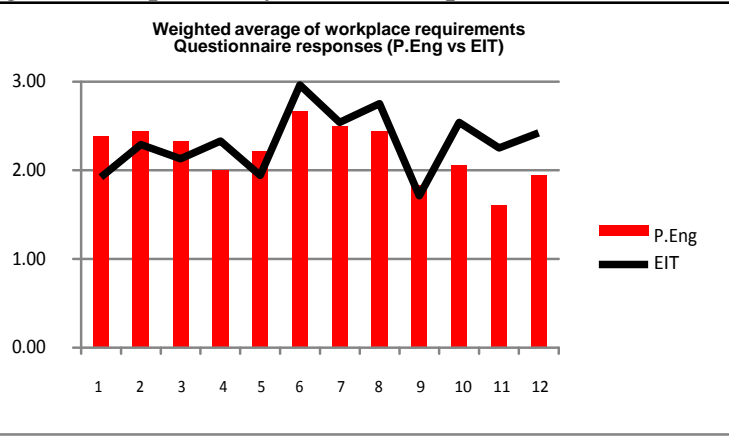

Figure 7: Comparison of graduate attribute questionnaire results between the professional engineers and engineers in training on new workplace requirements for new graduates.

Remarkably both groups rated the work requirements in a similar fashion. In the technical attributes 1Knowledge Base for Engineering, 2-Problem Analysis, 3-Investigation, and 5-Engineering Tools where the EIT's indicated their skill/abilities were adequate, they also rated the requirements lower than the P.Eng's. Both groups rated the attributes of 6-Individual and Teamwork, 6-Communication, and 7-Professionalism as having the highest requirements. These are the attributes that the EIT's discussed in the interviews as being the ones they felt most unprepared for upon entry to the workplace. The P.Eng's also discussed in the interviews that new graduates were required to have a high ability to communicate and work with others. The last three attributes 10-Ethics and Equity, 11-Economics and Project Management, and 12-Lifelong Learning shows the largest gap between the two groups. The EIT participants rated these fairly high and indicated in the interviews that they had trouble understanding what these 
entailed. The P.Eng group had a better understanding and said that these are areas new graduates will learn with experience and so rated them lower.

\subsection{Results of Ranking the Graduate Attributes}

The final data collected from the participants on the graduate attribute questionnaire was a ranking of the attributes. The rankings based on work requirements were very different than that of the rankings by competency level. In the ranking of new graduate competency levels both groups saw the technical levels as being the highest skill/ability level. The job requirement rankings showed that both groups strongly indicate the attributes 6Individual and Teamwork, 7-Communication, and 8Professionalism were the most important. The other attributes had more varied rankings with the P.Eng's finding the technical ones as important and the EIT's showing these with lesser value. This again goes back to discussions on the security EIT's felt with areas they were able to understand versus the unknown of what was expected in other areas.

\section{CONCLUSIONS}

The objective of this study was to identify the knowledge requirements of the workplace with respect to the CEAB graduate attributes. The information was compiled through the interviews and questionnaires from both P.Eng's and EIT's on the activities of newly graduated engineers.

The interview data had some limitations as discussions centered on certain graduate attributes with minimal data in the other attributes. Data was coded into the six levels of Blooms Taxonomy for each of the graduate attributes. Analysis of the interview data showed that the both the EIT and the P.Eng participants discussed the role of a new graduate as being in the comprehension and application levels of the graduate attributes. Assessment was consistent between the two groups in both the level of Bloom's Taxonomy and category of graduate attribute.

The second method of collecting information was with a questionnaire. The P.Eng's were asked to evaluate the capabilities and the job requirements for a newly graduated engineer. The EIT's were asked to give a self evaluation of their own capabilities at graduation and an assessment of their first job activities. The evaluation of the data showed consistency among the members of the groups and between the groups. This study focused on the job requirements of a new graduate based on the graduate attributes. The P.Eng group assessed the graduate attributes 6-Individual and Teamwork, 7Communication, and 8-Professionalism as the three top attributes for knowledge requirements for new graduate engineers. These three attributes were also assessed as having the greatest knowledge deficit for a new graduate with respect to the job requirements. The attributes of 2Problem Analysis and 4-Design were considered to have slight deficits in knowledge as require by new graduates with all other attributes requirements met by the knowledge of a new graduate. The EIT's classified themselves as having large knowledge deficits in the graduate attributes 6-Individual and Teamwork, 7Communication, and 8-Professionalism, and 11Economics and Project Management which closely matches the ratings by the P.Eng group. EIT's were critical of certain areas they felt they lacked knowledge required for their assigned activities. Other areas of concern for the EIT's were in 4-Design, 10-Ethics and Equity and 12-Lifelong Learning. The interesting information is that both groups felt that the highest requirements for the workplace were in the social aspects. Knowledge areas of communication, teamwork, and professionalism were classified as having the highest knowledge requirements along with the highest deficit in the knowledge attained by a new engineering graduate.

These would be important not only to a new engineering graduate but also to the supervisory role a P.Eng undertakes. This would be an area of concern and considered a gap in the knowledge required by a new graduate. The EIT's had an adequate knowledge base in the other graduate attributes, especially the technical areas. Since these areas were not shown to be important areas of responsibilities for the P.Eng participants would indicate that they are not considered to be a gap in knowledge for a new graduate engineer.

This study was on four professional engineers and four engineers in training within one corporation. This small sample size means that the conclusions are of a general nature and will be used as a guide for future research.

\section{References}

[1] Jill S. Cicek, Paul Labossiere and Danny Mann. "Surveying fourth year engineering student perceptions of graduate attribute competencies." In Proc of CEEA Canadian Engineering Education Conference, (Montreal, QC. June 2013)

[2] Engineers Canada, Graduate attributes, Accreditation Criteria and Procedures 2011, Canadian Engineering Accreditation Board, Accreditation Criteria and Procedures, Section 3.1 page 12. http://www.engineerscanada.ca/files/w_Accreditation_ Criteria_Procedures_2011.pdf 